REVISTA 89.1 Revista RELACIONES INTERNACIONALES Escuela de Relaciones Internacionales. Universidad Nacional, Costa Rica. N. ${ }^{\circ} 89.1$ • Enero-Junio de 2016 doi: http://dx.doi.org/10.15359/ri.89-1.3

\title{
RELACIONES INTERNACIONALES Y COMERCIO INTERNACIONAL: MÁS QUE UNA CORRESPONDENCIA ACADÉMICA, UN TRATO HISTÓRICO
}

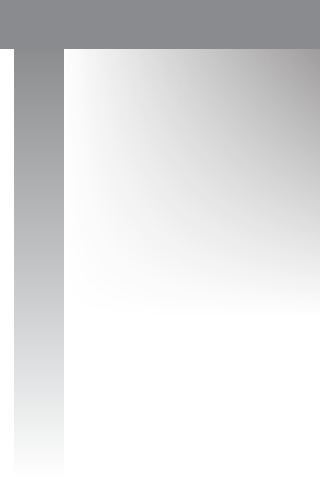

\author{
Daniel López Zúñiga*
}

\section{RESUMEN:}

Un tema que se ha prestado para la discusión académica en Costa Rica y Latinoamérica en los últimos años ha sido la pertinencia del estudio de temáticas económicas y comerciales en las escuelas de relaciones internacionales. Por esto, se tiene una necesidad de realizar una revisión del origen mismo de nuestra profesión. En este documento se hace una descripción del manejo temático dado al comercio internacional en relaciones internacionales, de manera que se pueda comprender su vínculo politológico, hurgando, incluso, en su objeto material y su objeto formal, que nos refiere a la antropología misma. También, se busca demostrar con evidencias históricas y antropológicas del parentesco entre relaciones internacionales y comercio internacional, tomando como parámetro los negocios entre centros de poder y reflexionando sobre algunos autores que nos permiten revelar dicho origen. Finalmente, se aborda la diplomacia y su difuso vínculo con el comercio internacional, tema tendencial de la integración como un nodo que nos remite a la esencia de la diplomacia y a comprender, así, la correspondencia académica de relaciones internacionales y comercio.

Palabras clave: Relaciones internacionales, comercio internacional, diplomacia económica, integración regional, negocios internacionales.

\begin{abstract}
:
Something that has been given to academic discussion in Costa Rica and Latin America in recent years has been the relevance of the study of economic and trade issues in the schools of International Relations. That is why you have a need for a review of the origin of our profession itself. This document provides a description of the subject management that has given the International Trade in International Relations, so that they can understand their political link, delving even in its material and formal object refers us to the same object becomes Anthropology. It also seeks to demonstrate historical and anthropological evidence of the relationship between International Relations and International Trade, on the parameter business between power centers and reflecting on some authors that allow us to reveal that source. Finally, diplomacy and diffuse inter trade link with the issue of integration trend as a nodule which refers to the essence of diplomacy and understanding academic correspondence of International Relations and Trade will be addressed.
\end{abstract}

Keywords: International Relations, International Trade, Economic Diplomacy, Regional Integration, International Business

\footnotetext{
* Máster en Relaciones Internacionales con énfasis en Cooperación Internacional. Ex académico de la Escuela de Relaciones Internacionales de la Universidad Nacional de Costa Rica y actualmente Primer Secretario en la Embajada de Costa Rica en Austria. Correo electrónico: daniellopezcr@gmail.com
} 


\section{Introducción}

Científicamente, se ha asociado el estudio de relaciones internacionales con temas referentes al juego de fuerzas entre unidades políticas o Estados, lo cual no es incorrecto, sino que es el enfoque que puede distorsionar la academia con respecto a la realidad, pues tras cada relación de cooperación y conflicto actual; se puede asociar al dilema sobre la posesión de recursos, intenciones de aumentar la interdependencia, cooperar para enfrentar los amplios mercados producto de asociaciones comerciales.

Por eso, el presente artículo iniciará con una conceptualización de relaciones internacionales y el carácter comercial que tradicionalmente ha tenido en la academia, independientemente de sus momentos de preeminencia o inferioridad. Además, se explicará cómo aspectos comerciales determinantes en las nociones de poder y, por ende, en la incidencia en el concepto de "continuum de las Relaciones Internacionales" dado por Barbé (2003, p. 218) para referirse a la cooperación y el conflicto de los centros de poder o Estados.

Ya entendido lo anterior, se seguirá con una vinculación histórica y antropológica de los aportes de los negocios a las relaciones internacionales y su fuerte atadura con la diplomacia; pero con un fuerte vínculo de diplomacia económica y comercial, vistas como determinantes de las manifestaciones de las relaciones entre los centros de poder, tomando como fundamento el Acuerdo de Kadesh.

Finalmente, se explicarán los conceptos de diplomacia económica y comercial como tema urgente en la dinámica internacional actual, y se concluirá con una demostración de que las relaciones internacionales no se están tornando primeramente comerciales, sino que pareciesen estar retornando a su esencia histórica.

\section{Comercio internacional en las relaciones internacionales}

Desde la génesis de las relaciones internacionales como una disciplina académica, se ha tenido una clara inclinación temática hacia las coyunturas políticas y las estructuras que estas han ido conformando a lo largo de la historia (Caritas Española 2012), pero no debemos olvidar las causas que llevaron, a los universitarios británicos en 1918 y luego a los estadounidenses en 1919, a constituir aquella derivación de la politología, y que fue precisamente el status quo de las relaciones entre Estados, o sea, a la dicotomía entre la guerra y la paz (Aron 1985). 


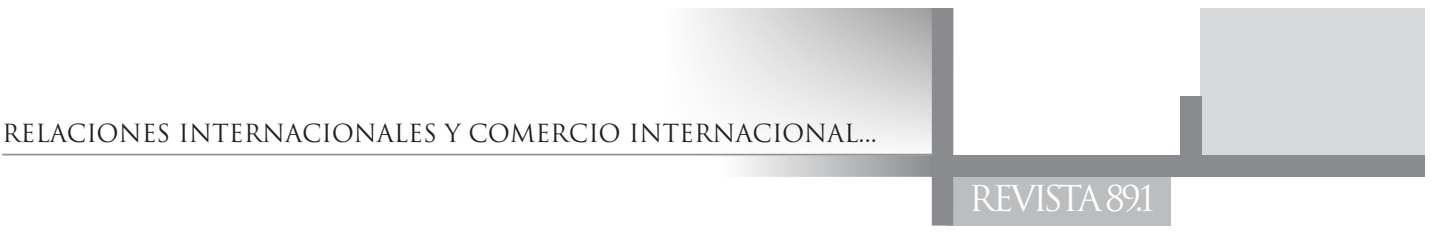

De ese primer planteamiento dialéctico se infirió que el proceso de las relaciones internacionales es de carácter bidimensional, en tanto que se expresan en términos de cooperación y conflicto, entendiendo la cooperación como cualquier interacción conducente al apoyo entre actores internacionales y, por el contrario, el conflicto se refiere a cualquier interacción que implica el choque de intereses entre actores internacionales, obviando la temática propia de la disciplina (Barbé 2003).

Fue en una obra que ha calado entre los pioneros internacionalistas, Guerras y paz entre las naciones, del profesor Raymond Aron (1985, p. vii), donde se da una definición particular del objeto formal de la disciplina, la cual dice que:

Quizá podría denominarse sociedad internacional o sociedad mundial al conjunto que engloba el sistema interestatal, la economía mundial (o el mercado mundial o el sistema económico mundial), los fenómenos transnacionales y supranacionales, aplicándose el adjetivo de internacional a todos los aspectos que he distinguido.

Derivado de esa definición, se puede afirmar que los temas comerciales se encuentran en la situación diferida de la organización política y de las ventajas naturales de los grupos humanos, que "territorializaron" el poder por aspectos meramente existenciales y de supervivencia, tal y como se ilustra en la figura 1. En otras palabras, y desde una perspectiva antropológica, las personas encontraron más fácil el sobrevivir en grupo que individualmente, lo cual indujo a formar organizaciones para garantizar la satisfacción de necesidades elementales (alimentación y protección del entorno).

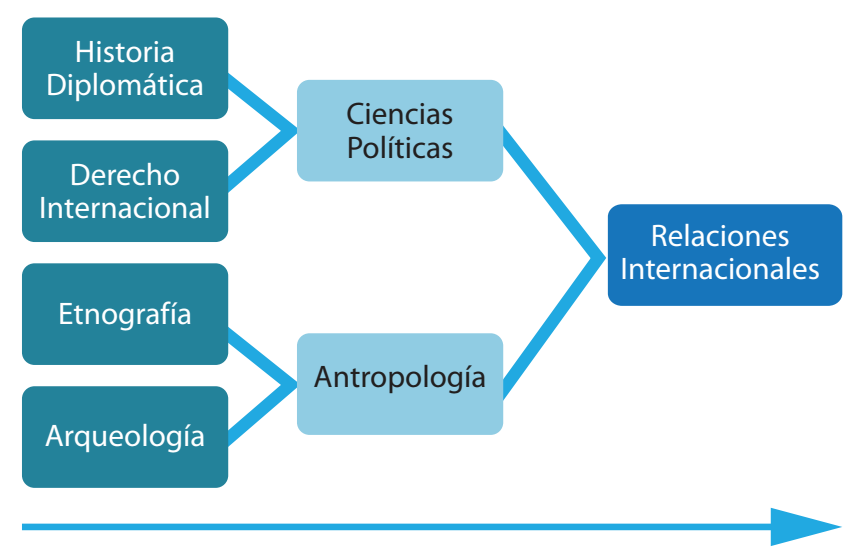

Figura 1. Origen científico de las relaciones internacionales.

Elaboración propia con datos de Barbé (2003) y Enguiguren (1987). 
Una mejor manera de comprender la prueba ofrecida por la antropología es que, en el caso de la alimentación, esta necesidad se solucionaba con el dominio de los recursos naturales; mientras que la protección del entorno únicamente se alcanzaba mediante el reconocimiento del paisaje y de sus amenazas, lo que implicaba la delimitación del espacio; así, se hizo imperioso el manejo de los recursos, con independencia de la etapa organizativa en que un grupo se encontrase (Lewellen 1994).

\section{a. Dominio del espacio y los recursos como componentes de poder}

En la misma línea de la evolución antropológica y política, se achaca al número demográfico y la especialización de funciones sociales el descubrimiento de la producción y, por ende, de la sedentarización (Enguiguren 1987). Fue precisamente con ese hito histórico cuando se materializó el poder, es decir, se "territorializó" (se ubicó en el espacio) y se "jurisdiccionó" (se establecieron normas en ese espacio).

Por lo anterior, se destaca que el advenimiento de la productividad llevó a la separación de funciones en la productividad del colectivo, lo cual indica que las tareas estarían asignadas a cada persona, según la hermandad y la filiación; esto con el objeto de maximizar la supervivencia de quienes se organizaban (Lewellen 1994). Así, nace el trabajo que le indicaría al individuo la dedicación a una tarea y en un espacio con un objeto para el colectivo, lo que conlleva la aparición de la propiedad privada.

Tomando referencias en las ciencias exactas, Isaac Newton, con su llamada "Ley de Impenetrabilidad" plantea que "dos cuerpos no pueden ocupar un mismo espacio en un momento determinado" (Sepúlveda 2003, p. 83), lo que ilustra que quien domina ciertos recursos naturales necesarios para vivir, por el hecho de ubicarse en un espacio dado, excluirá de su disfrute a otros ajenos a su colectivo. Lo anterior coincide con los planteamientos sobre la escasez que ha emitido la economía por muchos años y que antropológicamente explica la repartición del espacio terrestre entre grupos humanos que, luego, constituirán centros de poder encargados de intentar el dominio de recursos para su subsistencia; así aparece el conflicto (véase figura 2). 

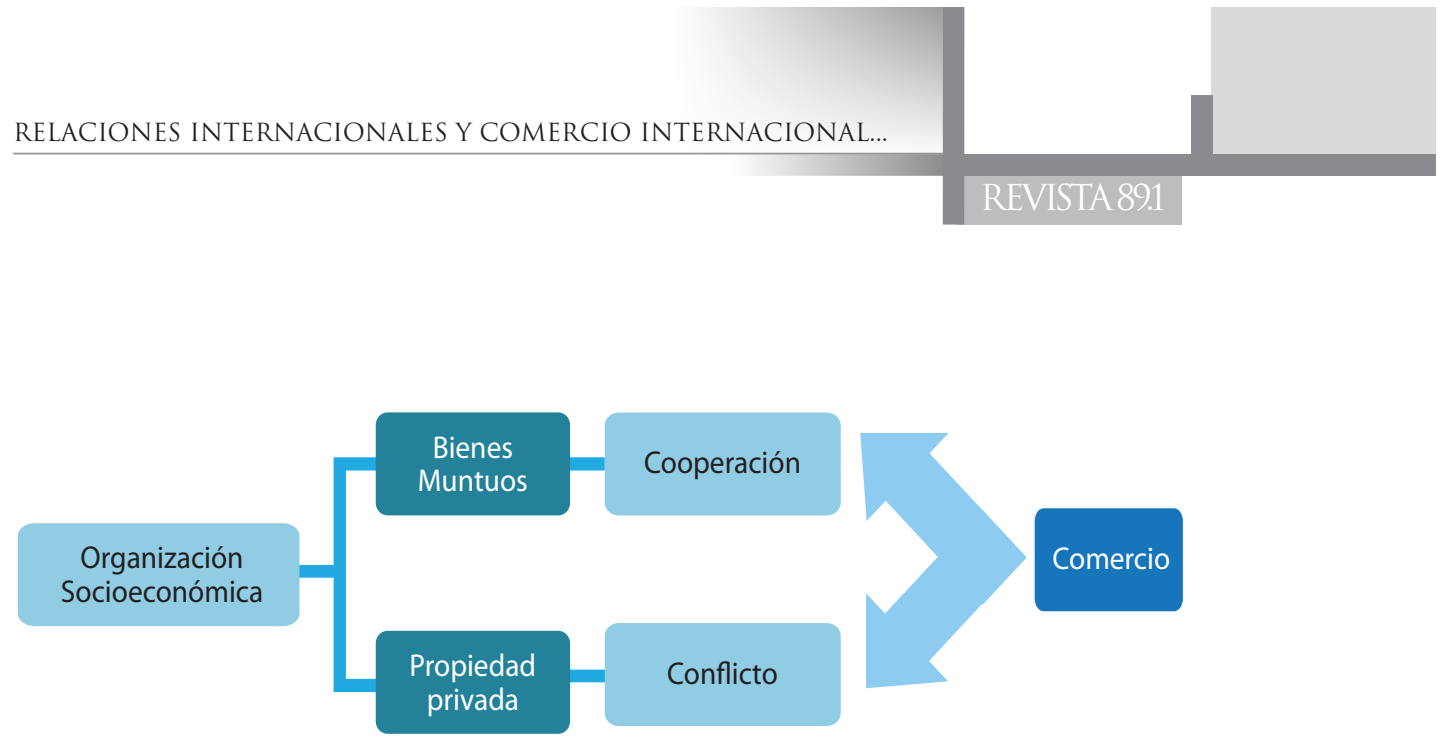

Figura 2. El comercio en el continuum de las relaciones internacionales. Elaboración propia con datos de Barbé (2003) y Lewellen (1994).

Las guerras entre tales centros de poder o unidades políticas (por no mal llamarlos Estados) llevaron a conclusiones oportunas que el propio Aristóteles manifestó en su obra La Crematística y que tiempo después se denominaría como el principio económico de las ventajas mutuas (Martínez-Echeverría y Ortega 2011), en el que se entrevé que es más oportuno para el objeto común (la supervivencia), lo cual en términos simples se explicaría como que un grupo humano aporte al otro su excedente productivo y viceversa; aparece así la cooperación.

\section{b. Indicios del comercio como manifiesto de cooperación}

Partiendo de los planteamientos aristotélicos, el comercio entre unidades de poder puede ser entendido en el continuum de las relaciones internacionales como una primera forma de cooperación y hasta de "diplomacia", tal y como se evidenció en la Batalla de Kadesh (hoy Siria) y su consecuente tratado en 1259 o 1274 a. C. ${ }^{1}$, donde hititas ${ }^{2}$ y egipcios solucionaron de manera bélica el dominio del principal centro comercial de la época, describiéndose así (Pirenne 1963, p. 238):

Punto de encuentro, cruce y negociación del tráfico y comercio de su tiempo, y área dotada de inconmensurables recursos naturales, Siria era la encrucijada mercantil, cultural y militar del mundo antiguo. No sólo producía ingentes cantidades de trigo, sino que por allí pasaban las mercancías provenientes de

1. Puede estudiarse la controversia sobre la fecha de este hecho en innumerables textos históricos de relaciones internacionales y en el mismo internet. El Tratado de Kadesh fue plasmado en tablones de arcilla y su original puede apreciarse en el Museo de Arqueología de Estambul, Turquía.

2. También llamados hetitas o heteos. 
los buques que cruzaban el Egeo y los de lugares más lejanos, que llegaban al Asia Menor por el puerto de Ugarit, especie de Venecia antigua que dominaba el comercio del Mediterráneo oriental, y se encontraba, precisamente, ubicada en Siria. Los derechos aduaneros que percibiría quien dominase la región eran enormes; sumados a su estratégica posición militar, la producción agropecuaria y los derechos de tráfico y exportación, convertían a la zona en una de las de mayor importancia estratégica del mundo antiguo.

La relevancia de Kadesh en la época se da porque en la zona se transportaba vidrio, cobre, estaño, maderas preciosas, joyas, textiles, alimentos, artículos de lujo, productos químicos, loza, porcelana, herramientas y metales preciosos, ya que la mayoría de las rutas comerciales se encontraban en ese sector debido a que de allí se mercadeaba a Medio Oriente y se recibían bienes desde Irán y Afganistán, como se conocen en la actualidad (González 2010).

Pero, independientemente de la importancia geopolítica de la propia Kadesh, el tratado que pone fin a dicha situación conflictiva es considerado como la evidencia más antigua de un acuerdo de paz entre unidades políticas, lo cual indica que el comercio será la vía de "bien seguir" para tales asuntos y así expresado en una cláusula de lo que aún se conserva: "El Gran Soberano del País de Hatti no entrará nunca en el País de Egipto para tomar cosa alguna. Y Userma Ra Setenpe Ra no penetrará en el País de Hatti para tomar cosa alguna" (Los escakeados, 2007, p. 4).

A partir de tales demostraciones se puede expresar que en el continuum de las relaciones internacionales se presenta el conflicto y la cooperación. Esta última se ubica en el comercio entre unidades políticas diferenciadas, definido como la anuencia aristotélica de proveer lo excesivamente producido por una unidad política y la demanda de la insuficiencia productiva de otra semejante.

\section{Diplomacia y negocios internacionales}

Con los avances demográficos y tecnológicos, la productividad en los centros de poder aumentó y dio lugar a una división simultánea e incipiente del trabajo a lo interno y externo, bajo una especialización ofrecida por las ventajas naturales de cada una, lo que conlleva a un aumento de la comercialización y de la búsqueda de utilidades sobre dicha actividad, más aún cuando aparece el dinero.

La tendencia por la que tanto los colectivos como los individuos buscan obtener un beneficio de su producción, más que encaminarse al capitalismo y 


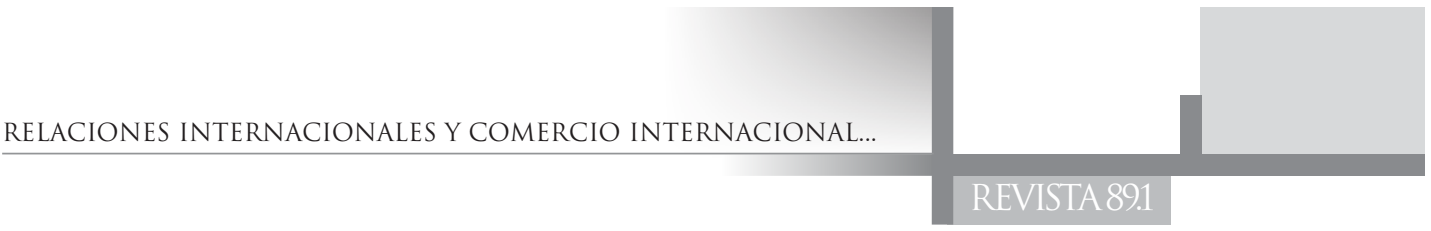

sus dilemas, conlleva al concepto de negocio. Un negocio es definido como el obtener un provecho o lucro por un bien o servicio comerciado (RAE 2001), mientras que de manera acertada, en el libro "Diplomacia" de Kissinger (2010, p. 40), define la Diplomacia como un "negocio en el que las partes son los Estados".

Por su parte, para John D. Daniels, Lee H. Radebaugh y Daniel P. Sullivan (2010), el acto repetitivo y de intención lucrativa entre los centros de poder, haría de los negocios sus interacciones y la diplomacia sus relaciones, por lo que "los negocios internacionales consisten en todas las transacciones comerciales (incluidas ventas, inversiones y transporte), que se llevan a cabo entre dos o más países" (Daniels, Radebaugh, \& Sullivan 2010, p. 86).

A pesar de haber descrito su origen formal en el intercambio de bienes y factores de producción, la evolución histórica de las interacciones entre centros de poder y el acaecimiento de los Estados, hizo que la cooperación política internacional, la seguridad y la preservación de la paz predominasen sobre la mera distribución de recursos naturales existenciales y el intercambio estratégico para la generación de utilidades tanto para el colectivo como para los individuos (Azuelos 2010). Lo anterior indica que el objeto material de la diplomacia mantuvo agendas paralelas en las que unas han sido más evidentes que otras, es decir, no han sido excluyentes; pero, con el tiempo, las temáticas se solaparon.

El nódulo entre diplomacia y negocios internacionales se comprende cuando se define el comercio internacional como un instrumento de desarrollo de los Estados, los cual nos inserta en el concepto de cooperación en relaciones internacionales (Barbé 2003) y, según Prado (2006, p. 532), este adopta el rango de "fuente de financiamiento del desarrollo" a como se ilustra en la tabla 1. 
Tabla 1.

Financiamiento internacional del desarrollo

\begin{tabular}{|c|c|c|c|}
\hline TIPO & PRIVADO & MIXTO & PÚBLICO \\
\hline 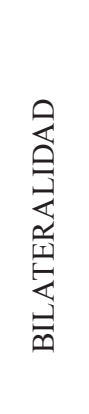 & $\begin{array}{l}\text { 1. Comercio internacional } \\
\text { 2. Inversión extranjera } \\
\text { 3. Créditos (bancarios o de } \\
\text { exportación) } \\
\text { 4. Remesas } \\
\text { 5. Turismo }\end{array}$ & $\begin{array}{l}\text { 1. Organizaciones no } \\
\text { gubernamentales } \\
\text { 2. Empresas } \\
\text { 3. Particulares } \\
\text { 4. Institutos } \\
\text { de investigación } \\
\text { 5. Global Enve- } \\
\text { rioment Facility } \\
\text { (GEF) }\end{array}$ & $\begin{array}{l}\text { 1. Agencias guberna- } \\
\text { mentales de coopera- } \\
\text { ción } \\
\text { 2. Miembros } \\
\text { CAD-OCDE } \\
\text { 3. Ayuda al desarrollo } \\
\text { 4. Cooperación para } \\
\text { el desarrollo } \\
\text { 5. Auxilio } \\
\text { en emergencias }\end{array}$ \\
\hline 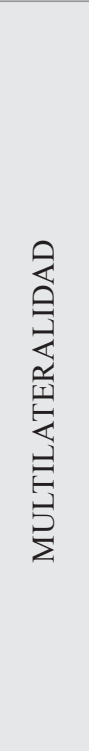 & $\begin{array}{l}\text { 1. Comercio regional o } \\
\text { multilateral } \\
\text { 2. Créditos bancarios, mer- } \\
\text { cados financieros, bonos } \\
\text { y obligaciones }\end{array}$ & $\begin{array}{l}\text { 1. Propuestas de } \\
\text { financiación de } \\
\text { bienes públicos } \\
\text { globales } \\
\text { 2. Impuestos Tobin, } \\
\text { energéticos, car- } \\
\text { bono, turbosina, } \\
\text { venta de armas, } \\
\text { etc. }\end{array}$ & $\begin{array}{l}\text { 1. Organismos } \\
\text { financieros } \\
\text { 2. Grupo del Banco } \\
\text { Mundial } \\
\text { 3. Bancos regionales } \\
\text { de desarrollo } \\
\text { 4. Bancos subregionales } \\
\text { de desarrollo } \\
\text { 5. Organismos } \\
\text { no financieros } \\
\text { 6. Sistema de } \\
\text { naciones unidas } \\
\text { 7. Comisión Europea } \\
\text { (Europe Aid) } \\
\text { 8. Fondo Europeo } \\
\text { de Desarrollo } \\
\text { 9. Banco Europeo } \\
\text { de Inversiones }\end{array}$ \\
\hline
\end{tabular}

Nota: Adaptación de Prado (2006).

La diplomacia económica (ver figura 3) se define como la "utilización de la capacidad de influencia política de los Estados en favor de sus intereses económicos en los mercados internacionales" (Morillas 2000, p. 42), por lo que se tendría cuatro grandes áreas temáticas de este tipo (Kishan 2000, p. 94): 
i. La promoción del comercio

ii. La promoción de la inversión

iii. La atracción de tecnología

iv. La ayuda al desarrollo.

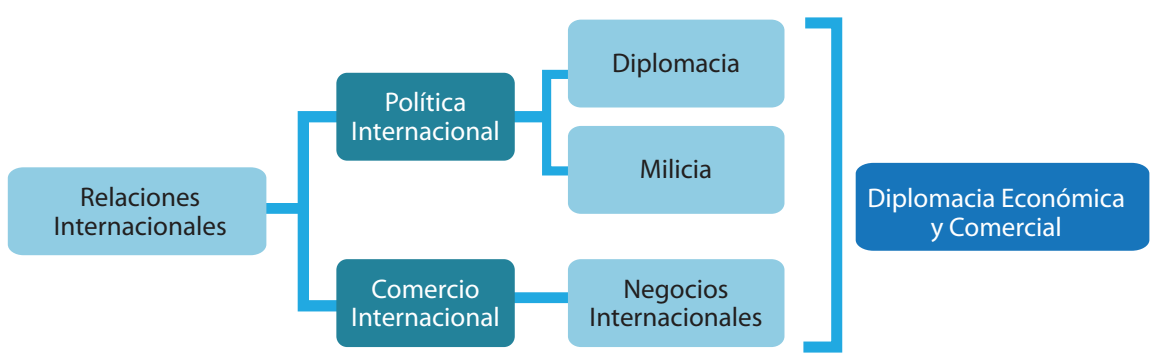

Figura 3. Origen científico de las relaciones internacionales.

Elaboración propia con datos de Morillas (2000) y Kishan (2000).

De lo anterior se podría inferir que la diplomacia comercial es aquella que se concentra en la promoción del comercio; pero, a partir de lo señalado por Kissinger, se puede ampliar este concepto a toda aquella actividad de negocios conformada por los Estados. Claramente, al aceptar esta significación de negocios se presumiría que los Estados tienen como objetivo el lucro, lo que en relaciones internacionales se reconocería como "Financiamiento del Desarrollo" (Prado, 2005, p. 532), específicamente en el impulso del intercambio de bienes, servicios o factores de producción, las inversiones (Bayne y Woolcock, 2011) y hasta temas de "marca país" (Sánchez, 2013).

\section{La diplomacia económica y comercial hoy}

Como se aprecia en la figura 4, para entender el modus operandi de la diplomacia económica y comercial es necesario hacer referencia a la significancia de la cooperación entre centros de poder o Estados para la promoción del comercio, de la inversión y la formulación de estrategias de "marca país", lo cual muestra un fuerte componente del tema comercial entre los Estados. 


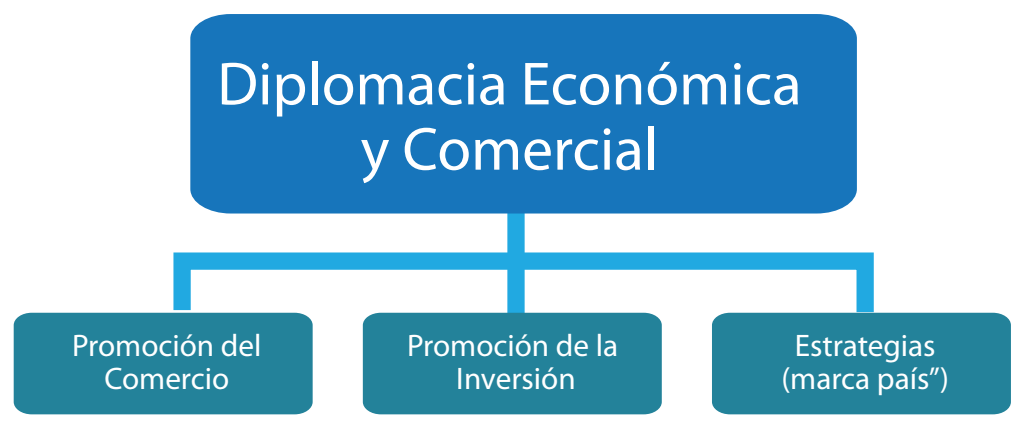

Figura 4. Temática de la diplomacia económica y comercial.

Elaboración propia con datos de Bayne y Woolcock (2011) y Sánchez (2013).

La integración económica corresponde a los acuerdos políticos y económicos entre países que concedan preferencias a los países miembros del acuerdo. Según Daniels, Radebaugh y Sullivan (2010), existen tres maneras en que la integración económica se puede comprobar según su alcance:

i. Integración bilateral (donde dos países deciden establecer relaciones de cooperación más estrechas, por lo general en forma de reducciones arancelarias).

ii. Integración regional (donde un grupo de países situados en la misma proximidad geográfica deciden cooperar).

iii. Integración global (la que se muestra en la Organización Mundial del Comercio $[\mathrm{OMC}])$.

Tal y como aconteció en Kadesh, hoy el modo de construir relaciones entre Estados es mediante la constitución de principios diplomáticos o "regímenes" que promuevan la cooperación y eviten el conflicto. La diplomacia comercial ha mostrado que cuanto más profundas y densas sean las relaciones de cooperación, mejor se tiende la construcción de procesos de integración económica que tienen como esencia el comercio.

\section{¿El retorno al origen de la diplomacia?}

Actualmente, el sistema internacional y las relaciones internacionales se concentran en el continuum de la cooperación, mientras que los conflictos se mantienen en una esencia territorial propulsada al dominio de los recursos. En otros 


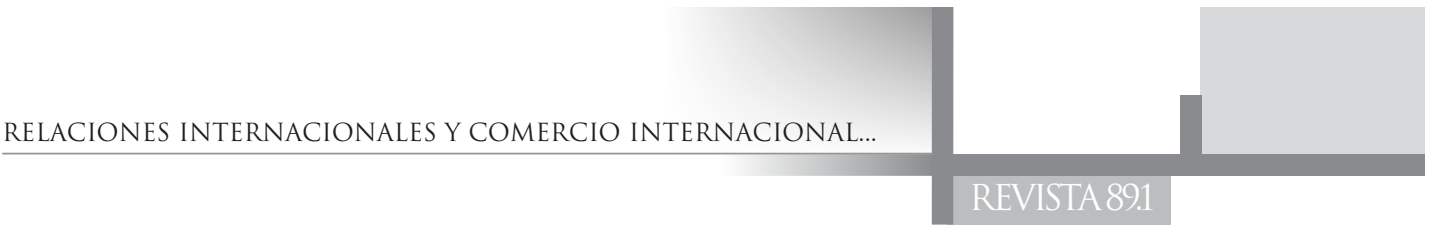

términos, la cooperación política internacional, la seguridad y la preservación de la paz dependen de la diplomacia económica y, específicamente, de la diplomacia comercial.

El regreso no se debe comprender como un retroceso al punto de partida, pues al avanzar se depende de la ruta elegida y, si esta es en forma de espiral o curva, perfectamente se tendrá la sensación de pasar por donde ya se ha estado. Se podrían enumerar gran cantidad de justificaciones para lo afirmado, pero se puede mencionar entre las más importantes (Sánchez Pérez-Castejón 2013, p. 350):

i. La integración financiera internacional impulsada por la liberalización del mercado de capitales y el desarrollo de los mercados financieros.

ii. La globalización de la mayor parte de mercados, lo que se ha traducido en el aumento de la competencia entre empresas y países.

iii. Complejización ante la aparición de nuevos competidores en forma de economías emergentes.

iv. Los avances en la Tecnología de la Información y la Comunicación que han facilitado, entre otras, la fragmentación de los procesos productivos.

La antropología, de acuerdo con Enguiguren (1987), nos insinúa que la cooperación lleva a que los colectivos conformen regímenes con aquellos semejantes que tuviesen interés existencial o estratégico, produciendo efectos casi imperceptibles en las relaciones internacionales durante mucho tiempo, entre ellos la creación del comercio y la desviación de este.

La creación de comercio se da cuando la producción o parte de ella se desplaza hacia productores más eficientes por razones de ventaja comparativa, lo cual lleva a la necesidad de relacionarse con ellos para aumentar la eficiencia, y la negociación de preferencias se convierte en el mecanismo para asegurar dichas relaciones. Por su parte, en la desviación del comercio este se desplaza hacia países del grupo, a expensas del comercio con otros que no pertenecen al grupo, pues resulta poco atractivo para las ínfulas de eficiencia (Daniels, Radebaugh, $\&$ Sullivan 2010). Ante esto, la tendencia ha sido la creación de comercio entre países situados en la misma proximidad geográfica: integración regional por medio del comercio. 


\section{La integración regional: Corolario de la actual diplomacia comercial}

Los cambios en la estructura y dinámica del sistema internacional en la década de los noventa hicieron inclinar la temática de las relaciones internacionales hacia la cuestión de los recursos, el abastecimiento y los mercados como solución a los problemas tanto nacionales como internacionales.

La tendencia inicial fue la conformación de acuerdos bilaterales que regularan los tratos preferenciales entre dos Estados, bajo el calificativo de acuerdos de comercio preferencial (ACP) ${ }^{3}$ o de tratados de libre comercio (TLC), con el objetivo de dinamizar las economías nacionales por medio del intercambio de bienes, servicios o factores de producción y, con ello, promocionar el desarrollo de las partes. Este tipo de tratados suele ser más sencillo de negociar para los países, que los propios acuerdos de la OMC, pues es menos complejo de entender y para comunicarse entre las partes.

Sin embargo, derivado de esta tendencia, la acepción de bilateralidad se volvió confusa, pues en la gran parte de ellos son dos Estados los que dominan la actoralidad de esos acuerdos, por ejemplo, Australia y Estados Unidos, Costa Rica y México, Cuba y Panamá. Situación particular de la actoralidad se apreciaría cuando esos tratados se suscribieron entre un Estado y otros (un bloque) apadrinados en un tratado anterior.

El caso más interesante se evidencia cuando la Unión Europea (UE) inicia posición a negociar cualquier tema comercial como bloque ante otros países, como se vivió en el Acuerdo de Asociación Comercial entre UE y Centroamérica, el cual es técnicamente bilateral, mientras que en el TLC entre Estados Unidos, Centroamérica y República Dominicana (CAFTA-DR), a pesar de ser siete países parte, estos negociaron de modo coordinado (Daniels, Radebaugh, \& Sullivan, 2010).

Poco a poco, la acepción de regional se ha ido arraigando en esta tendencia, pues la aparición de "negociaciones en bloque" incentiva asimetrías de intereses y de volúmenes productivos (capacidades), ante lo cual el objetivo primario de los más pequeños ha sido aumentar el tamaño del mercado.

Desde las propuestas de la antropología política y la historia económica que se describieron antes, se reafirman propuestas económicas de que la mejor manera de aumentar el tamaño del mercado es mediante la cooperación entre países.

3. Término empleado por Daniels, J. D., Radebaugh, L. H. y Sullivan, D. P. (2010, p. 300). 


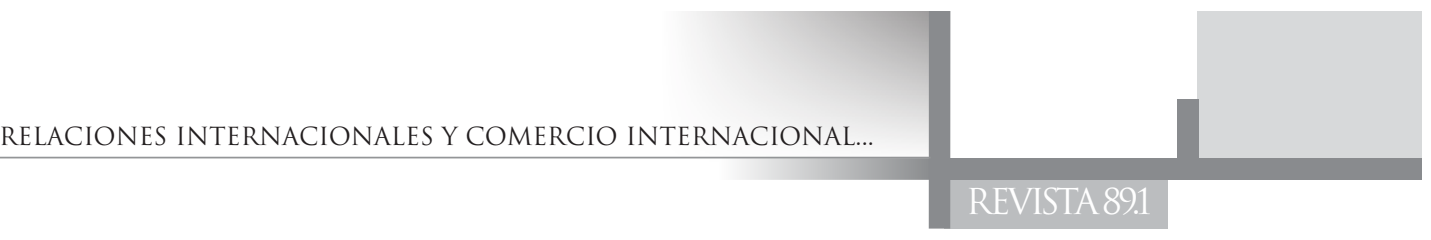

Dicha cooperación se ha impulsado a través de la constitución de zonas de libre comercio (sin aranceles internos), uniones aduaneras (sin aranceles internos y con aranceles externos comunes) y mercados comunes (unión aduanera más movilidad de los factores).

Sin embargo, Ghemawat (2001, p. 138) indica que existe una serie de condiciones básicas para facilitar tales estrategias de cooperación en el aumento del mercado, entre ellas:

a. Ubicación: que los gastos de transporte no distorsionen los precios de venta hacia compradores finales, por lo que lo ideal es la vecindad o cercanía entre países.

b. Cultura: los países pueden tener historias semejantes, intereses comunes, creencias, tradiciones e incluso estar más dispuestos a coordinar más fácilmente con unos que con otros.

c. Imaginario: la neuroeconomía indicaría la similitud en los gustos y preferencias de los consumidores, lo cual facilita los canales de producción y distribución en países vecinos.

Es por eso que se puede afirmar que existe una gama de acuerdos comerciales que poseen un alcance regional, es decir, Tratados Regionales de Comercio (TRC), en los que participan más de dos Estados que comercialmente son parte de una zona de libre comercio, una unión aduanera o un mercado común; todo ello, manifestaciones de cooperación en las relaciones internacionales, gracias a la diplomacia económica y, mayormente, a la comercial, las cuales, conforme se consolidan, dan lugar a la integración regional.

\section{Conclusión}

El comercio internacional se ha convertido en una disciplina medular para las relaciones internacionales de hoy, en tanto que cada tema, hecho o fenómeno que se desee estudiar lleva a su umbral de intercambio de bienes, servicios y factores de producción en una secuencia de recursos-soberanía-poder $\mathrm{y}$, precisamente cuando se alcanza la esencia real de las interacciones internacionales (Volz, 2011), cuando el conocimiento de esta área temática se vuelve fundamental en dicha carrera.

Cuando se estudia interdisciplinariamente el origen científico y académico de las relaciones internacionales se encuentra una serie de referencias de diversa 
índole que nos remiten a la historia diplomática y el derecho internacional para dar explicación a los mismos y, precisamente, al asimilar ambas disciplinas encontramos evidencias muy obvias de lo que hoy se denomina comercio internacional; disciplina que en épocas pasadas se limitaba al mero intercambio de bienes entre diferentes centros de poder o unidades políticas preestatales.

Al igual que apreciamos históricamente con los hechos de Kadesh, muchos gobiernos han emprendido una estrategia de profundización en el enfoque económico y comercial para el establecimiento de sus intereses ante el sistema internacional, lo cual ha engendrado una necesidad repentina de la diplomacia económica y, más aún, la comercial. Pareciese que lo que en la antigüedad causó diferendos, no difiere mucho de las controversias acontecidas en Ucrania, y que muchas acciones de política y comercio exterior se refieren a exportación, importación y medios de pago de bienes y servicios entre Estados, a saber: acuerdos preferenciales, tratados de libre comercio, alianzas de participación en bloques regionales y globales, rubros que no son incompatibles con estrategias de seguridad, cooperación política o ayuda al desarrollo.

Las relaciones entre organizaciones humanas, tanto antes como después de la aparición de los Estados, siempre han tenido un componente materialista por la posesión de recursos por el mero aspecto de la supervivencia, en otras palabras, se pueden tomar diferentes caminos, pero hay un umbral único; la supervivencia (o más recientemente, se pueden tomar diferentes caminos, pero el objetivo es uno: el desarrollo).

Podríamos decir que la fuerte inclinación por los temas económico-comerciales dentro de las relaciones internacionales de hoy ha requerido de una readaptación del quehacer diplomático tradicional, pues los intentos por separar la política comercial externa de la política exterior evidencian, en la práctica, que son más fuertes sus vinculaciones disciplinarias e históricas que sus disgregaciones en la práctica. 


\section{Referencias}

Aron, R. 1985. Paz y guerra entre las naciones. Madrid: Alianza Editorial.

Azuelos, M. (2010). Les évolutions de la diplomatie économique américaine. En L'age d'or de la Diplomatie Économique. Géoeconomie (pp. 2-19). París: Hiver.

Barbé, E. (2003). Relaciones internacionales. Madrid: Tecnos.

Bayne, N. y Woolcock, S. (2011). What is Economic Diplomacy. En L. S. Science, The New Economic Diplomacy. Decision-Making and Negotiation in International Economic Relations (pp. 1-21). Burlington: Ashgate.

Caritas Española. (2012). De la coyuntura a la estructura. Los efectos permanentes de la crisis. Madrid: Caritas.

Daniels, J. D., Radebaugh, L. H. y Sullivan, D. P. (2010). Negocios internacionales. Ambientes y operaciones. México D.F.: Pearson Education.

Enguiguren, J. E. (1987). Relaciones internacionales: Una perspectiva antropológica. Santiago, Chile: Editorial Andrés Bello.

Ghemawat, P. (Setiembre de 2001). Distance Still Matters: The Hard Reality of Global Expansion. Harvard Business Review , 3-11.

González, J. M. (2010). El Imperio Hitita. Suppiluliuma. Madrid, España: Editorial Aldebarán.

Kishan, S. R. (2000). Inside Diplomacy. Nueva Delhi: Manas Publications.

Kissinger, H. (2010). Diplomacia. Barcelona, España: Ediciones B, S. A.

Lewellen, T. (1994). Introducción a la antropología política (J. Aubet Semmler, Trad.) Barcelona, España: Edicions Bellaterra 2000.

Los escakeados. (2007). Comentarios del acuerdo entre Hattusil III y Ramsés II. Madrid: Jommia.

Martínez-Echeverría y Ortega, M. A. (2011). Técnica y crematística en Aristóteles. Revista Empresa y Humanismo , 14(2), 69-88.

Morillas-Gómez, J. (2000). La diplomacia económica. La otra mediación del Estado en el mercado. Boletin Económico de ICE, pp. 41-44.

Pirenne, J. (1963). Historia de civilización del antiguo Egipto. Barcelona: Éxito.

Prado, J. P. (2005). La condicionalidad politica de la cooperación al desarrollo: Las sanciones a la ayuda internacional (Tesis Doctoral). Somosaguas, Madrid, España: Universidad Complutense de Madrid. 
RAE.(2001). Diccionario Real Academia Española. Recuperado de Real Academia Española: http://lema.rae.es/drae/?val=negocio

Sánchez, P. (2013). La diplomacia comercial en el centro de la diplomacia económica española. Anuario Jurídico y Económico Escurialense , 345-362.

Sepúlveda, A. (2003). Los conceptos de física. Evolución histórica. Medellín, Colombia: Editorial de la Universidad de Antioquia.

Volz, U. (2011). Regional Integration, Economic Development and Global Governace. Northampton, Massachusetts, USA: Edward Elgar Publishing. 